OPEN ACCESS

Edited by:

Angel Angelov,

Tübingen University Hospital,

Germany

Reviewed by:

Jorge Frias-Lopez,

University of Florida, United States

Zhi-Luo Deng,

Helmholtz Association of German

Research Centres (HZ), Germany

*Correspondence:

Michelle M. Meyer

m.meyer@bc.edu

${ }^{\dagger}$ Present address:

Nikhil Ram-Mohan,

Department of Emergency Medicine,

Stanford University, Stanford, CA,

United States

Specialty section:

This article was submitted to

Systems Microbiology,

a section of the journal

Frontiers in Microbiology

Received: 18 November 2019

Accepted: 05 March 2020

Published: 09 April 2020

Citation:

Ram-Mohan N and Meyer MM

(2020) Comparative

Metatranscriptomics of Periodontitis

Supports a Common Polymicrobial

Shift in Metabolic Function

and Identifies Novel Putative

Disease-Associated ncRNAs.

Front. Microbiol. 11:482.

doi: 10.3389/fmicb.2020.00482

\section{Comparative Metatranscriptomics of Periodontitis Supports a Common Polymicrobial Shift in Metabolic Function and Identifies Novel Putative Disease-Associated ncRNAs}

\author{
Nikhil Ram-Mohant and Michelle M. Meyer* \\ Department of Biology, Boston College, Chestnut Hill, MA, United States
}

Periodontitis is an inflammatory disease that deteriorates bone supporting teeth afflicting $\sim 743$ million people worldwide. Bacterial communities associated with disease have been classified into red, orange, purple, blue, green, and yellow complexes based on their roles in the periodontal pocket. Previous metagenomic and metatranscriptomics analyses suggest a common shift in metabolic signatures in disease vs. healthy communities with up-regulated processes including pyruvate fermentation, histidine degradation, amino acid metabolism, TonB-dependent receptors. In this work, we examine existing metatranscriptome datasets to identify the commonly differentially expressed transcripts and potential underlying RNA regulatory mechanisms behind the metabolic shifts. Raw RNA-seq reads from three studies (including 49 healthy and 48 periodontitis samples) were assembled into transcripts de novo. Analyses revealed 859 differentially expressed (DE) transcripts, 675 more- and 174 less-expressed. Only $\sim 20 \%$ of the DE transcripts originate from the pathogenic red/orange complexes, and $\sim 50 \%$ originate from organisms unaffiliated with a complex. Comparison of expression profiles revealed variations among disease samples; while specific metabolic processes are commonly up-regulated, the underlying organisms are diverse both within and across disease associated communities. Surveying DE transcripts for known ncRNAs from the Rfam database identified a large number of tRNAs and tmRNAs as well as riboswitches (FMN, glycine, lysine, and SAM) in more prevalent transcripts and the cobalamin riboswitch in both more and less prevalent transcripts. In silico discovery identified many putative ncRNAs in DE transcripts. We report 15 such putative ncRNAs having promising covariation in the predicted secondary structure and interesting genomic context. Seven of these are antisense of ribosomal proteins that are novel and may involve maintaining ribosomal protein stoichiometry during the disease associated metabolic shift. Our findings describe the role of organisms previously unaffiliated with disease and identify the commonality in progression of disease across three metatranscriptomic studies. We find that although the communities are diverse between 
individuals, the switch in metabolic signatures characteristic of disease is typically achieved through the contributions of several community members. Furthermore, we identify many ncRNAs (both known and putative) which may facilitate the metabolic shifts associated with periodontitis.

Keywords: periodontitis, oral microbiome, metatranscriptomics, non-coding RNA, riboswitch, antisense

\section{INTRODUCTION}

Afflicting more than three million individuals in the United States per year, periodontitis is an oral disease characterized by inflammation of the periodontium (resulting from poor oral hygiene) that eventually leads to tooth loss. Given the prevalence of periodontitis, the microbial instigators of the disease have been studied for decades, with novel technologies successively contributing toward a better understanding of the disease and its polymicrobial origins. Cultivation based methods originally identified anaerobic, Gram negative, rods deep in the periodontal pocket (dominated by Bacteroides melaninogenicus and Fusobacterium nucleatum) (Slots, 1977). Circumventing the limitations of culture-based techniques, $16 \mathrm{~S}$ rRNA sequencing from the subgingival plaques of 50 individuals with advanced periodontitis revealed eight key periodontal pathogens: Actinobacillus actinomycetemcomitans, Bacteroides forsythus (now Tannerella forsythia), Campylobacter rectus, Eikenella corrodens, Porphyromonas gingivalis, Prevotella intermedia, Prevotella nigrescens, and Treponema denticola (Ashimoto et al., 1996), and further screening expanded the list of periodontal pathogens to include members of Deferribacteres, Bacteroidetes, OP11, and TM7 phyla, and several novel species including Eubacterium saphenum, Porphyromonas endodontalis, Prevotella denticola, and Cryptobacterium curtum (Kumar et al., 2003).

A subsequent high-throughput DNA hybridization study of periodontitis progression in 185 individuals revealed six complexes of disease associated organisms characteristic of distinct stages of disease progression (red, orange, yellow, green, blue and purple) (Socransky et al., 1998). The red complex, comprised of Porphyromonas gingivalis, Treponema denticola, and Tannerella forsythia, colonizes the biofilm during late stage periodontitis and is the major pathogenic complex (Socransky et al., 1998; Ximénez-Fyvie et al., 2000; Holt and Ebersole, 2005). The orange complex, which is strongly associated with the red complex, is comprised of a larger number of species including - Campylobacter gracilis, C. rectus, C. showae, Eubacterium nodatum, Fusobacterium nucleatum, Parvimonas micra, Prevotella intermedia, Prevotella nigrescens, and Streptococcus constellatus. As colonization by the orange complex progresses, more members of the red complex also colonize, suggesting that during disease, colonization of the orange complex directly precedes the red complex (Socransky et al., 1998; Socransky and Haffajee, 2005). Members of the other four complexes (purple, blue, yellow, and green) partake in the initial colonization of the periodontal pocket causing a cascading effect leading toward the orange and red complexes (Socransky and Haffajee, 2005).
The advent of metagenomic analysis has further underscored that periodontitis is a result of polymicrobial synergy and dysbiosis (Lamont and Hajishengallis, 2015). The mean species diversity of the microbial community appears to change drastically between healthy and periodontitis affected states. However, the precise effect is not well understood. Initial 454-pyrosequencing of $16 \mathrm{~S}$ rRNA libraries followed by qPCR of 22 chronic periodontitis samples uncovered higher alpha diversity and biomass associated with the disease community. This finding suggests that new dominant taxa emerge, but the original health-associated community may not be replaced (Abusleme et al., 2013). However, subsequent meta-analysis across several studies shows reduced alpha diversity associated with disease (Ai et al., 2017). Based on fluctuations of relative metagenome abundances there appear to be a set of marker species that differentiate healthy, stable, and progressing sites of periodontitis (Ai et al., 2017). Furthermore, comparison of 16 metagenomic samples revealed the existence of a core disease affiliated community (Wang et al., 2013). However, the keystone pathogens of periodontitis that interfere with host immune defenses leading to tissue destruction [Porphyromonas gingivalis (Hajishengallis et al., 2011, 2012; Orth et al., 2011; Darveau et al., 2012), Prevotella nigrescens, and Fusobacterium nucleatum (Szafrański et al., 2015)] are not among the identified marker species that differentiate diseased and healthy samples. The observed changes in community structure go hand in hand with an alteration in the functional profile of the community. Metagenomic surveys of healthy and diseased dental plaques revealed that genes encoding bacterial chemotaxis, motility, and glycan biosynthesis and metabolism are over-represented in disease whereas metabolism of carbohydrates, amino acids, energy, and lipids, membrane transport, and signal transduction are under-represented (Wang et al., 2013).

Three metatranscriptomic surveys have provided further insight directly into metabolic activity during disease progression (Duran-Pinedo et al., 2014; Jorth et al., 2014; Yost et al., 2015). Gene ontology enrichment analyses of metatranscriptomes from progressing and stable periodontitis sites revealed that members of the red complex up-regulate their TonBdependent receptors, aerotolerance genes, iron transport genes, hemolysins, and CRISPR-associated genes, and enzymes like proteases and peptidases (Yost et al., 2015). However, transcripts (for processes such as proteolysis, potassium transport, and cobalamin biosynthesis) from organisms not previously associated with disease also show differential expression, suggesting involvement of additional organisms (Yost et al., 2015). Functional comparisons of healthy and aggressive periodontitis sites revealed that upregulation of lysine fermentation, histidine degradation, and pyruvate metabolism 
are common to the diseased individuals (Jorth et al., 2014). The collective finding of all three metatranscriptomic studies is the conservation of the community functionality rather than the specific microbial effecters of disease (Jorth et al., 2014; Duran-Pinedo et al., 2015; Yost et al., 2015). This finding emphasizes how understanding of the etiology of disease has progressed from the red complex instigators, to the keystone pathogen concept, and to finally toward a polymicrobial synergy and dysbiosis model (Hajishengallis and Lamont, 2012; Lamont and Hajishengallis, 2015).

While most of the emphasis of past metatranscriptomic analysis has been on identifying up-regulated protein coding regions and organisms associated with these coding regions, a functional bacterial transcriptome also includes many noncoding RNAs (ncRNAs). ncRNAs are untranslated, often structured, elements that are key posttranscriptional regulators acting on mRNA degradation (Desnoyers et al., 2013), translation initiation (Urban and Vogel, 2007; Frohlich and Vogel, 2009), synthesis of ribosomal proteins (Deiorio-Haggar et al., 2013), and transcription attenuation (Breaker, 2012) in response to environmental cues. These regulatory elements can be divided into cis-and trans-acting based on their location on the genome with respect to the regulated target. Riboswitches are classical examples of cis-acting ncRNAs that are typically found in the $5^{\prime}$-untranslated regions (5'UTR) immediately upstream of the regulated gene. ncRNAs are also found antisense to coding regions and often interfere with transcription (Neufing et al., 2001) or repress translation (Sayed et al., 2012). Only the metatranscriptomic study conducted by Duran-Pinedo et al. screened for differentially expressed ncRNAs in periodontitis. This study identified 20 Rfam families within a reported 12,097 small RNAs (sRNAs) overrepresented in disease (Duran-Pinedo et al., 2015). Activities regulated by these ncRNAs include: amino acid metabolism, carbohydrate metabolism, control of plasmid copy number, response to stress, and ethanolamine catabolism (Duran-Pinedo et al., 2015). However, Duran-Pinedo et al. defined sRNA very broadly as any transcribed, but not protein-coding, genomic region, and only screened for known ncRNAs [i.e., those in the Rfam database (Griffiths-Jones et al., 2003, 2005; Daub et al., 2008; Burge et al., 2013; Nawrocki et al., 2015)] to identify biologically relevant regions. Thus, it is likely that there are additional ncRNAs with biological function in the oral metatranscriptome associated with periodontitis progression that have not been well-described.

Despite decades studying periodontitis and its microbial instigators, the overarching mechanism of disease is still unknown. The organisms, underlying genes, and a short list of ncRNAs driving the disease have been assessed, but the underlying common mechanism of disease progression across the multitude of studies is yet unknown. The current study was undertaken as a meta-analysis to elucidate the genes and potential regulatory mechanisms common to the progression of periodontitis in patients across multiple studies and to illuminate the extent of variation in functional composition between individuals. We achieved this by combining existing RNA-seq read data from three previously published studies (Duran-Pinedo et al., 2014; Jorth et al., 2014; Yost et al., 2015) resulting in a total of 97 pooled metatranscriptome datasets (49 healthy and 48 diseased) to provide increased statistical support and detect subtle differences between the states and the studies (Gibbons et al., 2018). In contrast to previous studies, we also employed a de novo transcript assembly method rather than aligning the reads to a 'super genome' of the $\sim 400$ available oral genomes in the Human Oral Microbiome Database (HOMD) or limiting our analysis to only protein coding regions. In addition to screening for known non-coding RNAs, we also engaged a de novo discovery of RNA secondary structures in the assembled transcripts. Our analyses support findings from the earlier studies. We employed standardized cross-sample normalization and identified the common differentially expressed genes and known ncRNAs across 48 disease samples suggesting a shift in metabolic signatures during progression of periodontitis and identified many novel putative structured ncRNAs revealing the potential for riboregulation in periodontal disease.

\section{MATERIALS AND METHODS}

\section{Data Sources}

Previously published RNA-Seq data (Duran-Pinedo et al., 2014; Jorth et al., 2014; Yost et al., 2015) was compiled to result in a total of 49 healthy and 48 disease datasets. Individual fastq files may be found under BioProject accession number SRP033605 as Sequence Read Archives (SRAs) and under submission numbers 20130522 and 20141024 in the publication data repository of HOMD. The Yost et al., 2015 and Duran-Pinedo et al., 2014 data were collected on an Illumina MiSeq vs $2(2 \times 150$ or $2 \times 250$ cycle cartridges), the Jorth et al., 2014 data were collected as single-end 50 -bp reads on an Illumina HiSeq2000.

\section{Assembly and Annotation of Transcripts}

Trinity was run on the 97 read files using the -trimmomatic flag and default settings to assemble transcripts. Assembly quality was estimated using the auxiliary scripts as part of the Trinity software and included calculating the basic contig statistics, read representation of the assembly, and assessing the number of full length coding transcripts. Additional filtering of the assembled transcripts using Transrate (Smith-Unna et al., 2016) was performed to remove bad contigs. Coding regions within these transcripts were identified using the default settings of TransDecoder (Haas et al., 2013), which was also used for downstream processes such as annotation and binning of the intergenic regions. The assembled transcripts were then annotated two ways. First, the microbial source of each transcript was identified at the species level using BLAST (Altschul et al., 1990) against a local database of the HOMD annotated genomes. Second, the transcripts and identified coding regions were searched against a local Uniprot database using blastx and blastp, respectively, to determine protein functions. The transcripts were also screened for protein domains using hmmscan [from HMMER (Finn et al., 2011)] against the PFam database (Finn et al., 2014) and rRNA by running RNAMMER (Lagesen et al., 2007). Hits were filtered for e-value of less than 
$1 \mathrm{e}-10^{4}$ and the remaining hits were compiled into a SQLite boilerplate database to generate detailed annotation reports for each transcript using Trinotate (Haas et al., 2013). Alongside, the transcripts were also annotated using the KEGG Automatic Annotation Server (KAAS) (Moriya et al., 2007) to derive KEGG Orthology (KO) numbers.

Apart from the functional and taxonomic annotations of the transcripts, we also screened differentially expressed transcripts for known structured non-coding RNA (ncRNA). ncRNAs were identified in the transcripts by searching for every covariance model in Rfam12.2 (Griffiths-Jones et al., 2003, 2005; Daub et al., 2008; Burge et al., 2013; Nawrocki et al., 2015) using cmsearch from infernal-1.1.1 (Nawrocki and Eddy, 2013). Hits with an e value less than $10^{-4}$ were collected and all nonprokaryotic hits (e.g., U1 spliceosomal RNA and Histone3) were dismissed and the remaining ncRNA hits were included in the transcript annotations.

\section{Differential Expression and GO Term Enrichment Analyses}

All predicted rRNA transcripts were removed and the expression of the remaining transcripts was quantified in each dataset using Salmon (Patro et al., 2015). Salmon uses a two-phase method employing a quasi-mapping approach as opposed to a traditional alignment-based method to generate count data quickly. A combined matrix was then created for the healthy and disease states with the raw counts for transcript expression. The matrix of expression counts was imported into R (R Core Team, 2015) and differential expression analysis was carried out using edgeR (Robinson et al., 2009), the Bioconductor package. Briefly, the counts per million mapped reads $(\mathrm{cpm})$ were calculated for each dataset and low expression transcripts $(\mathrm{cpm}<0.5)$ in more than half of the datasets were removed. Since our interests lie in screening for the common effectors in the progression of the disease, we pooled all datasets in each state together (Healthy and Disease) and use each sample as a replicate to estimate common dispersals from the trend in expression. Next, cross sample normalizations using the trimmed mean of M-values (TMM) method was carried out. This normalized expression of transcripts in each sample against an arbitrarily chosen reference sample and excluded outliers. exactTest was run on the filtered transcripts between the healthy and disease states to identify the DE transcripts. Only transcripts with a log fold change $\left(\log _{2} \mathrm{FC}\right) \geq 1$ or $\leq-1$ with a FDR corrected $p$ value $<0.05$ were considered significant. Gene Ontology (GO) term enrichment analysis of the DE transcripts was carried out using GOseq (Young et al., 2010), the Bioconductor R package. Gene lengths, of the assembled transcripts, were estimated with a perl script that is a part of the Trinity installation. Enriched and depleted GO terms estimated in health and disease were considered significant if the FDR corrected $p$ value $\leq 0.05$. The DE genes were then mapped onto KEGG pathways to precisely locate the steps that were highly up- or down-regulated. These were visualized using Pathview (Luo and Brouwer, 2013, 2015), the Bioconductor package. Further, the major contributing species in each sample for the enriched processes or pathways was identified by surveying the individual metatranscriptome read files using HUMAnN2 (Abubucker et al., 2012).

\section{Functional Taxonomic Makeup of Disease Samples}

In order to establish the functionally active taxonomic makeup of the disease samples being studied, the metatranscriptomic reads were subject to MetaPhlAn2 (Truong et al., 2015), a part of the HUMAnN2 package for analyzing microbiome datasets (Abubucker et al., 2012). To determine the microbial community composition that is functionally active in each sample we mapped all the reads using Bowtie2 (Langmead et al., 2013) to a custom compiled marker sequence database available with HUMAnN2. Relative abundances were calculated to identify the prevalent species in each sample.

\section{Comparison of Expression Profiles Between Samples}

Based on the differential expression analysis, the significant transcripts were selected from the entire transcript collection and the expression profile in each of the 49 healthy and 48 disease samples were compared for these transcripts. Pearson correlation coefficients ranging between -1 for a negative relationship to 1 for a positive relationship were calculated for each pairwise observation using the cor function in $\mathrm{R}$.

\section{In silico Identification of Known ncRNAs and de novo Discovery of Putative Structured ncRNA}

In an effort to identify ncRNAs that might regulate the progression of periodontitis, the DE transcripts were assayed for both known and novel structured ncRNAs. Known ncRNAs deposited in Rfam12.2 (Griffiths-Jones et al., 2003, 2005; Daub et al., 2008; Burge et al., 2013; Nawrocki et al., 2015) were identified using cmsearch from infernal-1.1.1 (Nawrocki and Eddy, 2013). de novo discovery was performed by GraphClust (Heyne et al., 2012). The intergenic regions were pooled and the nucleotide sequences extracted. The compiled sequences were processed two ways -1 ) all of the compiled sequences together; 2) blast hits for the sequences against Refseq77 (O'Leary et al., 2016) were collected and the each set of hits was run separately. GraphClust, employing infernal-1.0.2 (Nawrocki et al., 2009); RNAshapes (Steffen et al., 2006); LOCARNA (Will et al., 2007, 2012; Smith et al., 2010); ViennaRNA (Lorenz et al., 2011); and RNAz (Gruber et al., 2010), was programmed to search for structures in sliding windows of 150 nucleotides with a shift of 75 nucleotides.

The predicted putative structures and their covariance models derived by GraphClust were searched against the entirety of the Refseq77 genomic database using an in house perl pipeline to obtain more, phylogenetically distant hits if possible. In short, the generated covariance model was converted to version 1.1.1 of infernal, calibrated and then searched against the sequence database. High confidence hits were extracted and realigned with the original covariance model. The resultant Stockholm file was then filtered to remove sequences that do not have at least $60 \%$ 
of the predicted structure before manual curation on RALEE (Griffiths-Jones, 2005).

\section{RESULTS}

\section{Only a Fraction of the Differentially Expressed (DE) Transcripts Originate From Red/Orange Complexes}

RNA-Seq data published by earlier studies (Duran-Pinedo et al., 2014; Jorth et al., 2014; Yost et al., 2015) that surveyed the community composition as well as the functional profile variation between healthy and periodontitis affected sites were collected. Despite many possible differences between the studies patient profile; disease severity; methodology used; we initially combined the datasets in an effort to identify the underlying commonality across all studies. We accumulated read data from a total of 49 healthy and 48 diseased samples. The collected data were split into the two categories - Healthy with a total of 26,034,228 reads, and Disease with 34,697,369 reads, and concatenated into a combined dataset for de novo assembly using Trinity (Grabherr et al., 2011; Haas et al., 2013). This generated a total of 627,752 transcripts with an average GC content of $49.76 \%$, a median contig length of 298 nucleotides, and an N50 of 420 nucleotides with an overall alignment rate of $84.67 \%$. Transrate filtering of these resulted in a total of 479,578 good contigs. Of these, 255,086 coding sequences were predicted using TransDecoder and $\sim 43 \%$ were annotated with a GO term.

Differential expression analyses of the transcripts between healthy and diseased states using edgeR with counts first normalized to each dataset and then cross-sample normalized resulted in a total of $859 \mathrm{DE}$ transcripts with a $p$ value $<0.05$ and a $\log _{2} \mathrm{FC} \geq 1$ or $\leq-1$ (Supplementary Table S1). Of these, 675 showed increased expression in diseased samples, and 184 displayed decreased expression. Although such differences are the result of both up- or down-regulated gene expression within an organism and frequency changes in members of the microbial community, we will refer to increased prevalence transcripts as up-regulated, and decreased prevalence transcripts as down-regulated throughout this manuscript. The transcripts are represented by 157 species and strains spanning 52 genera. Only $\sim 20 \%$ of the up- or down-regulated transcripts originated from the members of the red or orange complexes and $\sim 50 \%$ originate from microbes unaffiliated with any specific complexes (Figure 1A). Interestingly, many unaffiliated microbial species are closely related to those previously classified into the microbial complexes and show large counts of DE transcripts. For example, members of the genus Streptococcus account for 212 of the $859 \mathrm{DE}$ transcripts and span many of the disease associated complexes. S. constellatus is grouped in the orange complex; and S. gordonii, S. intermedius, S. mitis, S. sanguinis, and $S$. oralis are classified in the yellow complex. However, S. anginosus, S. australis, S. cristatus, S. infantarius, S. infantis, S. mutans, S. oligofermentans, S. vestibularis, S, pneumoniae, S. peroris, S. parasanguinis, and several Streptococcus oral taxa are unaffiliated with specific complexes yet still contribute significantly to the DE transcripts.

The up- and down-regulated transcripts originate from 121 and 63 bacterial species, respectively. The ten species with the largest number of up-regulated transcripts are shown on Table 1, and the majority are not affiliated with a disease associated complex. Members of the orange complex account for $\sim 18 \%$ of the up-regulated transcripts whereas red complex members account for only $\sim 4 \%$. Of the down-regulated transcripts, $\sim 24 \%$ and $16 \%$ are derived from Streptococcus sanguinis SK36 and Streptococcus gordonii Challis CH1, respectively, both of which are members of the yellow complex. Three transcripts from Treponema (red complex), and two from Fusobacterium (orange complex) are the only down-regulated transcripts from either complex, consistent with the large presence of these pathogenic bacteria, and the resulting increase in their transcriptome, in diseased samples.

\section{Differentially Expressed Transcripts From the Orange and Red Complexes Show the Greatest Magnitude Changes}

Although the red and orange complexes account for only a small percentage of the differentially expressed genes, the magnitude of change in expression in these transcripts is drastic (Figure 1B). The up-regulated red complex transcripts range from a $\log _{2} \mathrm{FC}$ of 3.6 to 8.2 with an average expression change of $\sim 6.1$-fold, and the down-regulated transcripts range from -5.8 to -7.4 -fold with an average drop in expression of $\sim 6.6$-fold. Similarly, the change in expression of the up-regulated transcripts from the orange complex ranges from $\sim 1.5$ to $\sim 7.9$-fold with an average change in expression of $\sim 4$.6-fold. Only two transcripts from the orange complex are down regulated with changes in expression of -2.5 and -2.3-fold. Members of the other complexes do not undergo such a drastic change in expression. Change in the expression of transcripts from the blue complex ranges from about 1.2 to 4.8 fold (average: $\sim 1.9$ ) and -1.9 to -4.5 -fold (average: -2.7 ); green complex ranges from 1.8 to 4.8 -fold (average: 3.5 ), with no downregulated transcripts; purple complex ranges from 1.4 to 3.9 -fold (average: 2.0), and two down-regulated transcripts with $\log _{2} \mathrm{FCs}$ of -2.9 and -2.6 , respectively; yellow complex ranges from $\sim 1.2$ to 4.4 (average: 2.1 ), and $\sim-1.2$ to -5.1 (average: -2.03 ). Finally, the unaffiliated group, with many diverse genera, displays changes in expression between $\sim 1.1$ to 7.4 -fold with an average change in expression of 2.6 -fold, and $\sim-1.5$ to $\sim-8.1$ with an average down-regulation of $\sim-3$-fold.

\section{Expression Profiles of Disease Samples Are Dissimilar}

To assess whether the expression profiles of DE transcripts of disease samples are similar to one another, correlation between profiles of individual samples was estimated. The Pearson correlation coefficient (PCC) was calculated for each pairwise comparison of samples. The PCC is a measure of the linear correlation between two variables where the resulting value between -1 and 1 suggests either a negative linear correlation or a positive one, respectively. From a heatmap of the correlation 


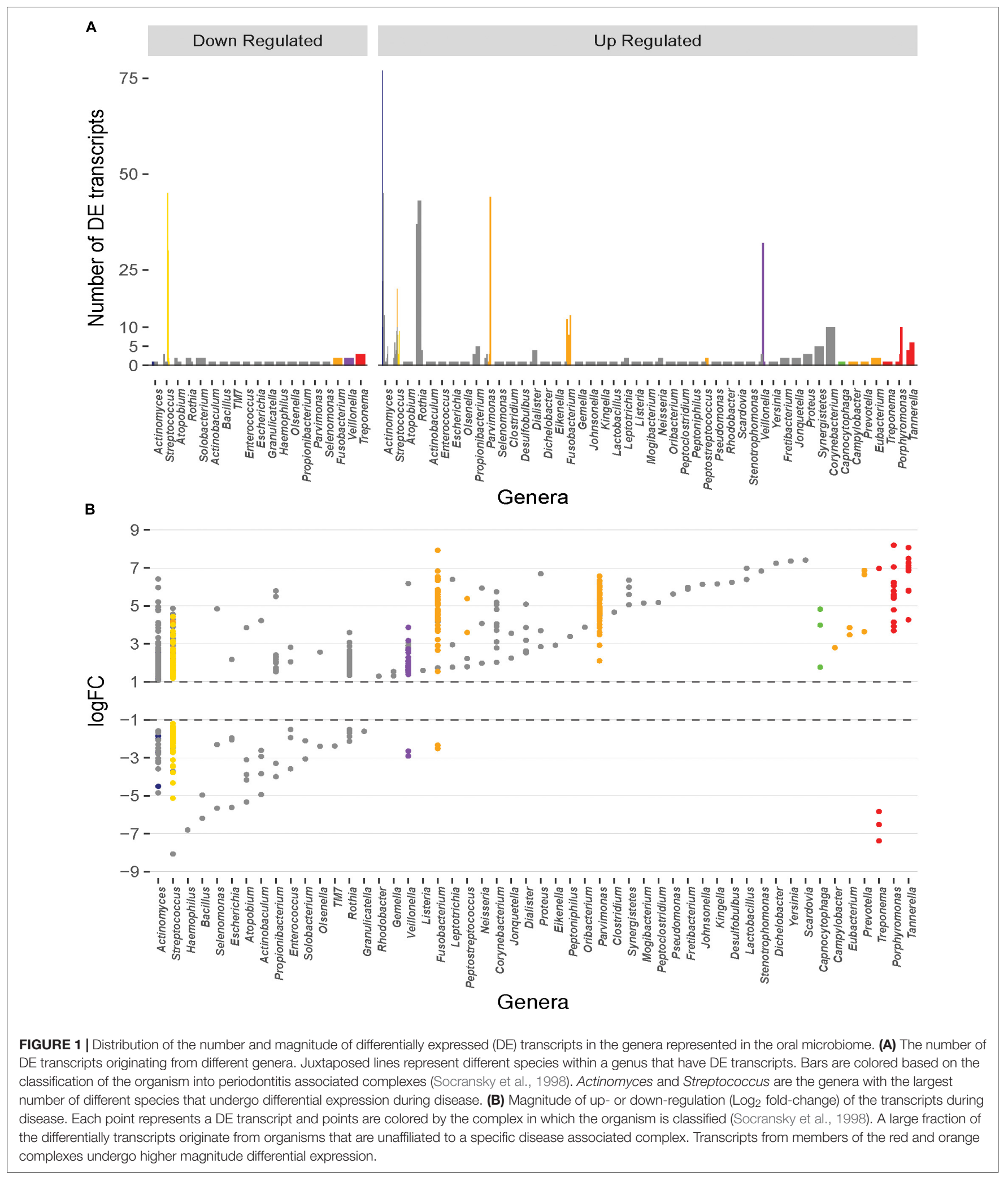

values (Figure 2), it is immediately apparent that there is no strong correlation between all the healthy samples. This is expected as the oral microbiome in each individual is likely to be highly complex and variant. There is also no strong correlation in the expression profiles of the disease samples. In fact, samples tend to cluster based on the study in which they were generated 
TABLE 1 | Species with the largest number of DE transcripts.

\begin{tabular}{llc}
\hline Organism & Complex & $\begin{array}{c}\text { \% of total up-regulated } \\
\text { transcripts }\end{array}$ \\
\hline Actinomyces viscosus C505 & Blue & $\sim 11 \%$ \\
Actinomyces oral taxon 175 & Unaffiliated & $\sim 7 \%$ \\
Parvimonas micra ATCC 33270 & Orange & $\sim 7 \%$ \\
Rothia dentocariosa M567 & Unaffiliated & $\sim 6 \%$ \\
Rothia dentocariosa ATCC 17931 & Unaffiliated & $\sim 5 \%$ \\
Veillonella parvula DSM 2008 & Purple & $\sim 5 \%$ \\
Actinomyces oral taxon 171 & Unaffiliated & $\sim 3 \%$ \\
$\begin{array}{l}\text { Streptococcus constellatus subsp. } \\
\text { pharyngis SK1060 }\end{array}$ & Orange & $\sim 3 \%$ \\
Fusobacterium nucleatum subsp. & Orange & $\sim 2 \%$ \\
vincentii ATCC 49256 & & $\sim 2 \%$ \\
Actinomyces oris K20 & Unaffiliated &
\end{tabular}

List of top 10 species with the most number of up-regulated transcripts. Includes information of the microbial complexes these organisms belong to.

in rather than the disease state. However, intra study clustering is also not absolute, there are instances where samples from different studies cluster together. This finding is consistent with the diverse criteria for sample inclusion, collection site, and methodology across the original studies.

The lack of correlation in the expression profiles can be attributed to the variation in the community composition of the samples. The functionally active genus and species level composition of each disease sample (using the entire metatranscriptomes) show distinct involved communities in each sample (Figure 3A). The differences are apparent not only in variations in the relative abundances of active members between communities, but also in the presence/absence of members of each genus and species. Assessing the top 50 most functionally abundant genera in the entire dataset (Figure 3A), only 17 genera are commonly found in $>50 \%$ of the samples (Figure 3B). Members of the Streptococcus genus were most frequently detected ( $~ 96 \%$ of the disease samples). Members of genera Rothia and Veillonella were the next commonly detected bacteria in the disease samples ( $92 \%)$. Despite their strong association with pathogenicity, members of the Porphyromonas, Tannerella, Fusobacterium, and Treponema genera were only detected in $\sim 65 \%, \sim 56 \%$, $\sim 52 \%$, and $\sim 52 \%$ of the disease samples, respectively. Similarly, at the species level, of the 100 most abundant species in the entire dataset, only 14 species were found in $>50 \%$ of the disease samples. Unlike the genus level, no single Streptococcus species is detected in a majority of the samples. However, Rothia dentocariosa, Actinomyces viscosus, Porphyromonas gingivalis, Tannerella forsythia, Veilonella parvula, and Fusobacterium nucleatum are well represented. The analyses also find highly active viruses in the periodontal pockets including Tobamovirus, Endornavirus, Gammaretrovirus, Potyvirus, and Mastadenovirus. It should also be noted that samples originating from Jorth et al., 2014 were pooled across multiple sites, while those from Yost et al. and Duran-Pinedo et al. originate from individual sites. This likely explain the apparent broad species representation in the 3 samples originating from Jorth et al. (Figure 3A). Thus,

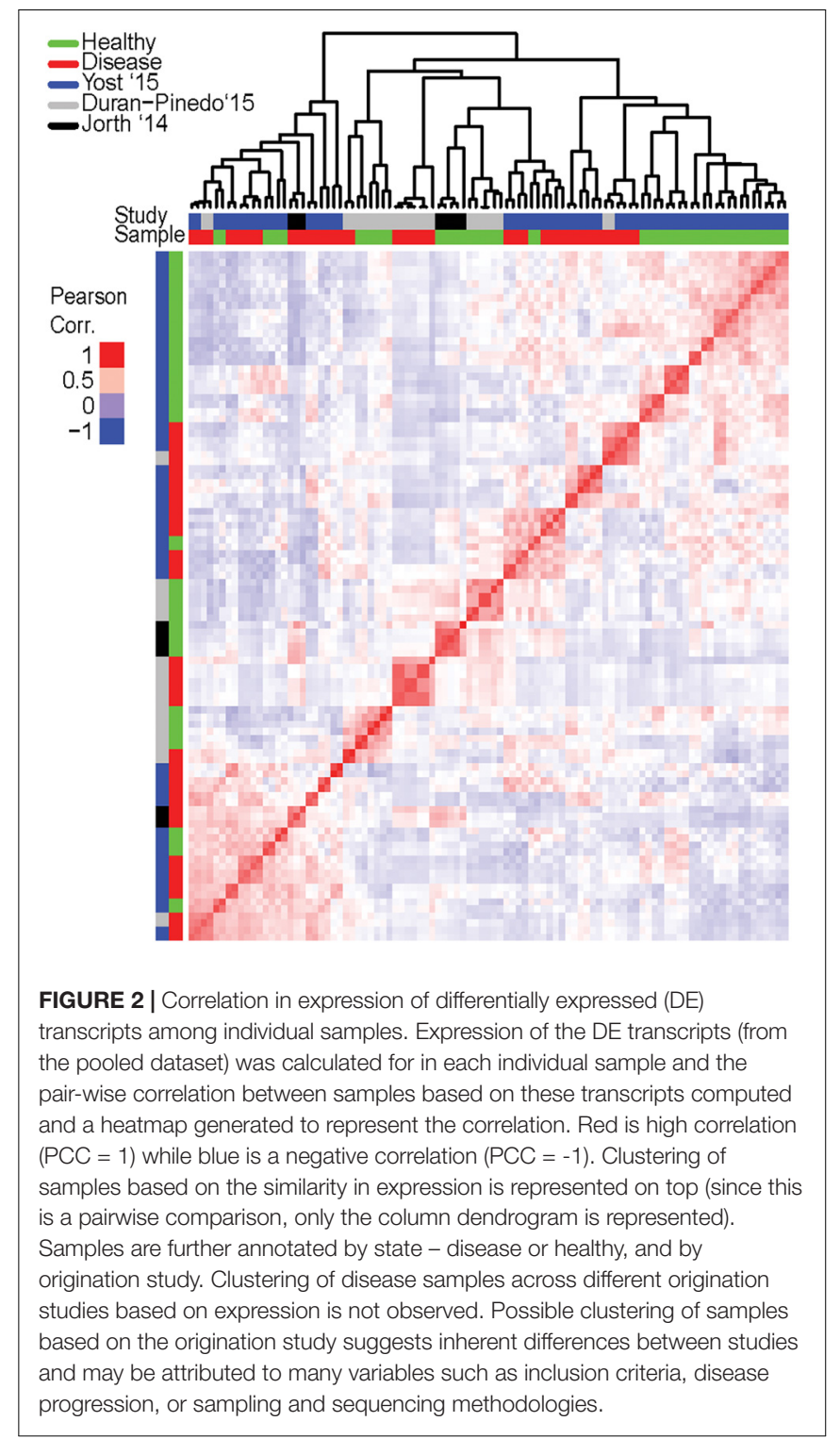

the discrepancy in correlation of expression of DE transcripts between disease samples is likely a result of the drastic variation in the functionally active taxa among samples, and is not likely purely due to differences between originating studies.

\section{Specific Metabolic Processes Are Enriched in Disease}

To identify the biological processes that are enriched in the DE transcripts, GO term enrichment analyses using GOseq was conducted. This analysis revealed that biological processes that involve only one organism are significantly less expressed in disease including lipid metabolic processes, hydrolase activity, and peptidase activity in individual organisms. The analyses also revealed an enrichment of genes categorized under the umbrella biological process of localization (e.g., transporter proteins, protein localization to cell surface). These are likely involved in establishment of pathogen localization in the pocket, 


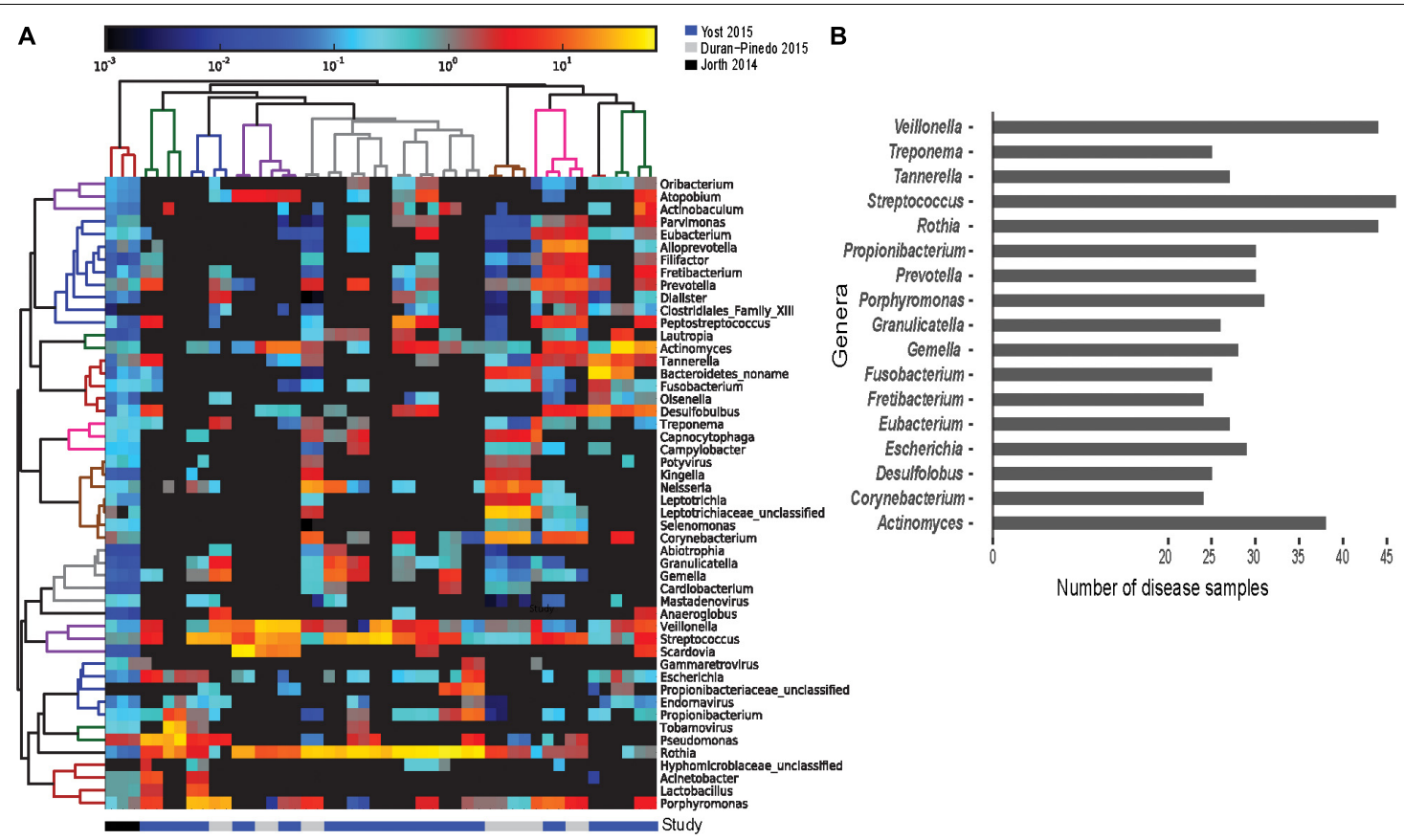

FIGURE 3 | Prevalence of functionally active taxa across disease samples. Screening for the functionally active microorganisms in the disease samples. (A) Heatmap of the 50 most functionally active genera from the overall dataset identified from the metatranscriptomes using HUMAnN2 (Abubucker et al., 2012). Gradient of color represents the abundance of the genus in a disease sample. Dendrograms represent hierarchical clustering of samples (column) and genera (rows) representing the similarity in the taxonomic composition between the samples and relative abundance of various genera, respectively. (B) Frequency distribution of the genera identified in at least 24 of the 48 disease samples. Streptococcus, Rothia, and Veillonella were found active in 40 out of the 48 disease samples. Porphyromonas, Tannerella, and Fusobacterium were found active in 30 samples or fewer.

as periodontitis progresses by the successive localization of various microorganisms in the pocket, until the members of the red complex arrive to drive pathogenesis. Other enriched processes include transport (cation, organic substance, nitrogen compounds, proteins, amino acids), biosynthesis (nitrogen compounds, aromatic compounds, and RNA), metabolism (catabolism of organic substances, glycolytic processes, pyruvate and amino acid metabolism), transcription, and translation.

\section{Metatranscriptomics Supports the Polymicrobial Nature of Periodontitis}

The polymicrobial nature of a periodontal infection is readily apparent in the diversity of microorganisms observed to be the sources of the DE transcripts. Mapping the DE transcripts on to KEGG pathways using Pathview revealed communities working in unison to provide a functional shift in disease. For example, mapping the increased prevalence transcripts on to the KEGG pathway for pyruvate metabolism (ko00620) showed both strongly and weakly increased expression genes in the pathway. Surprisingly, annotating these transcripts by their microbial sources revealed $\mathrm{DE}$ transcripts originating from members of several complexes (Figure 4). Different steps along the pathway were increased or decreased in prevalence within distinct organisms of the community.

To further assess this observation, the microbial contributors to the pyruvate pathway in each individual disease sample were identified using HUMAnN2. The variation observed in the species contributing to pyruvate metabolism in different samples is striking (Figures $\mathbf{5 A}, \mathbf{B}$ ). Up-regulation of genes involved in the fermentation of pyruvate to acetate and lactate were detected in 38/48 disease samples (Figure 5A). Other than four samples where Streptococcus tigurinus and two samples where Rothia aeria are the sole contributors in the pathway, the remaining 44 samples show a combination of various species contributing to the pathway, a community effort. Similarly, genes involved in the fermentation of pyruvate to isobutanol were up-regulated in 46/48 disease samples (Figure 5B). Again, other than the 8 samples that display a single organism as the sole contributor to this pathway - Streptococcus tigurinus (2 samples), Streptococcus mitis (2 samples), Rothia dentocariosa (4 samples), the remaining show a combination of organisms contributing to the pathway. This phenomenon is not unique to the pyruvate metabolism pathways. Similar patterns emerge when mapping the $\mathrm{DE}$ transcripts to the glycolysis/gluconeogenesis pathway, TCA cycle, lysine biosynthesis pathway, and butanoate metabolism to list a few. In contrast, upregulation of the one carbon pool by folate pathway is driven solely by the members of the orange complex, the zinc/manganese/iron transporters are up-regulated only in the members of the orange complex, and the fermentation of lysine to butanoate is achieved only by Fusobacterium nucleatum and Fusobacterium periodonticum in 17/48 disease samples. Nonetheless, most metabolic pathways that are enriched under disease conditions seem to have multiple contributing species 


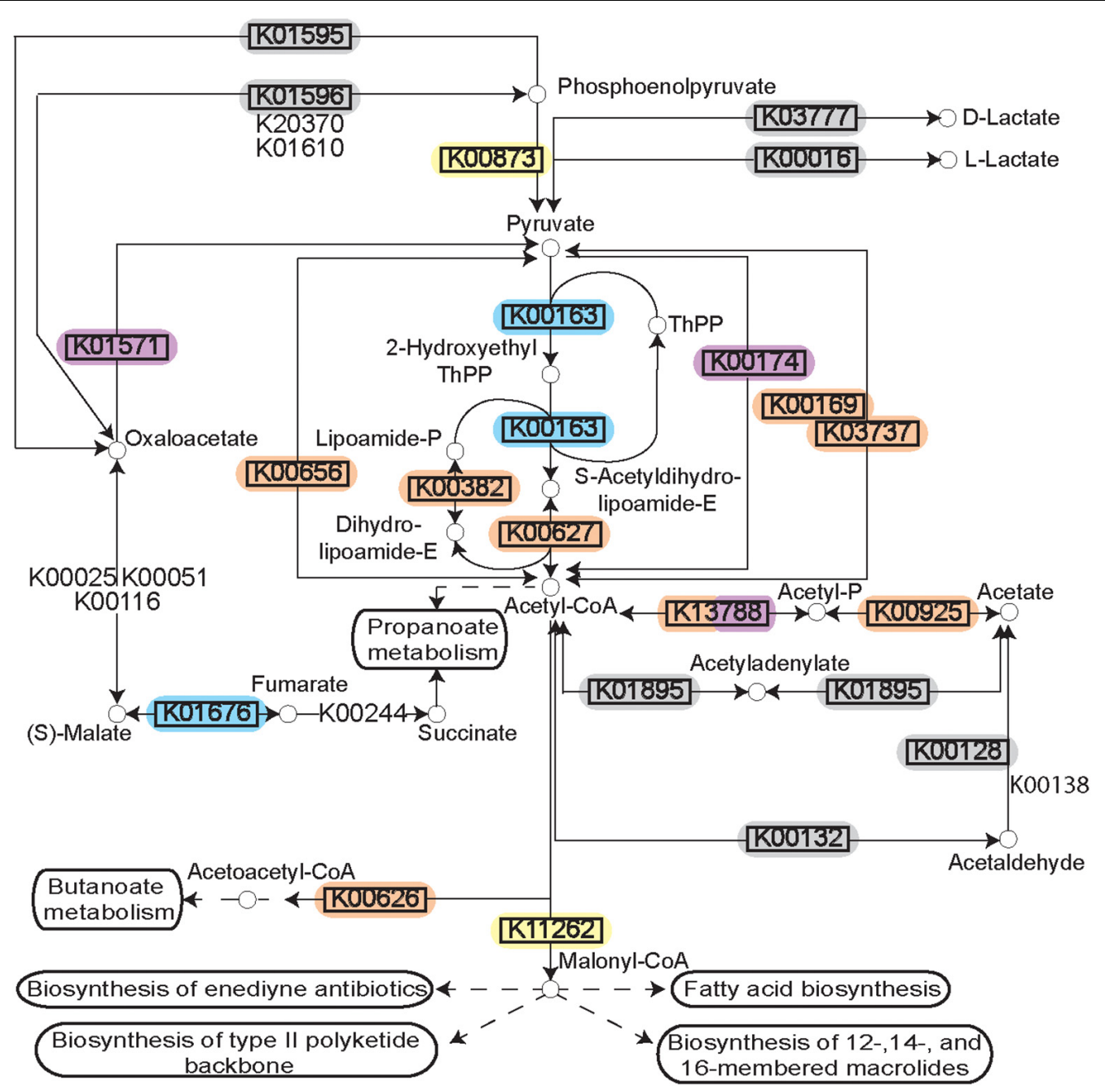

FIGURE 4 | Pyruvate metabolism during disease. Rendering of the KEGG pathway of pyruvate metabolism annotated with the DE transcripts and the microbial complexes from which they originate. Differential expression is represented by the black rectangle around the KEGG Orthology term. Background color for the KEGG orthology term represents the microbial complex of the organism that showed differential expression. The pathway as a whole is up-regulated, but the individual genes are up-regulated in diverse species supporting polymicrobial synergy during periodontitis.

fulfilling the metabolic niche within a single diseased sample, and different steps along the pathway show increased prevalence due to various contributory species.

\section{Known Structured Non-coding RNA Are Present in the DE Transcripts}

In an effort to identify possible regulatory mechanisms associated with disease, a screen for known ncRNAs in the DE transcripts was carried out using Infernal 1.1.1. This screen returned a total of 635 hits. All non-prokaryotic hits (e.g., U1 spliceosomal RNA, Histone3) were removed leaving 181 known structured ncRNAs in the assembled transcripts (Figure 6). The two predominant classes of ncRNA identified are tRNAs and tmRNAs, which constitute $41 \%$ and $19 \%$ of the identified ncRNAs identified. These are critical RNA molecules responsible for essential bacterial function, and display both up- and down-regulation depending on the organism. These differences may reflect a change in community members rather than a change in functionality of the community. In addition, the 6S RNA (constituting $3 \%$ of the ncRNAs identified) is up- or downregulated in various unaffiliated genera, and up-regulated in a member of the orange complex - the genus Eubacterium. There were also ncRNAs displaying only increased prevalence. RNase P bacterial classes A and B together represent $\sim 9 \%$ of DE ncRNAs. Class A RNaseP originates from members of the blue, orange, and red complexes and unaffiliated organisms, whereas class B only originates from unaffiliated members of genus Streptococcus, consistent with the phylogenetic distribution of the two RNase P classes. The bacterial small signal recognition particle (SRP) RNA ( 3\% of DE ncRNAs) is also only up-regulated, predominantly by members of the orange complex, namely - Fusobacterium, Parvimonas, and Peptostreptococcus. 
A

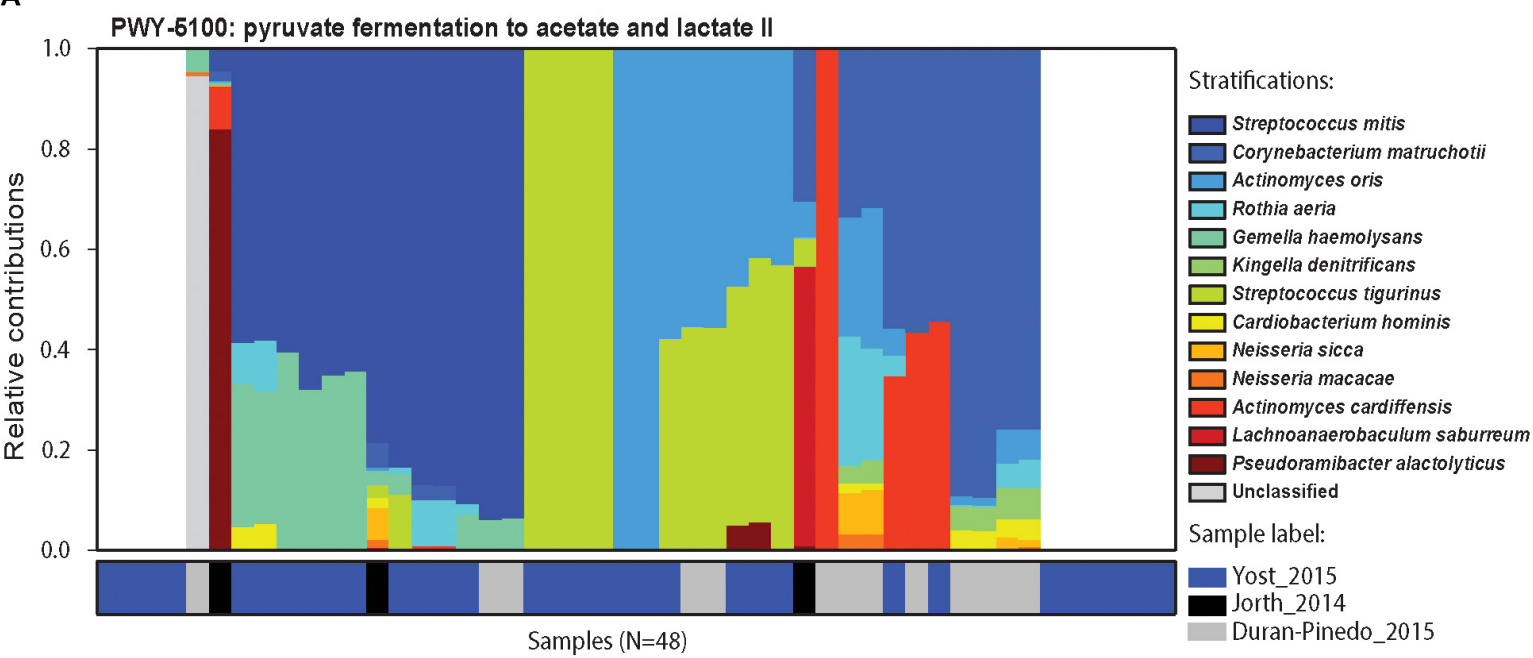

B

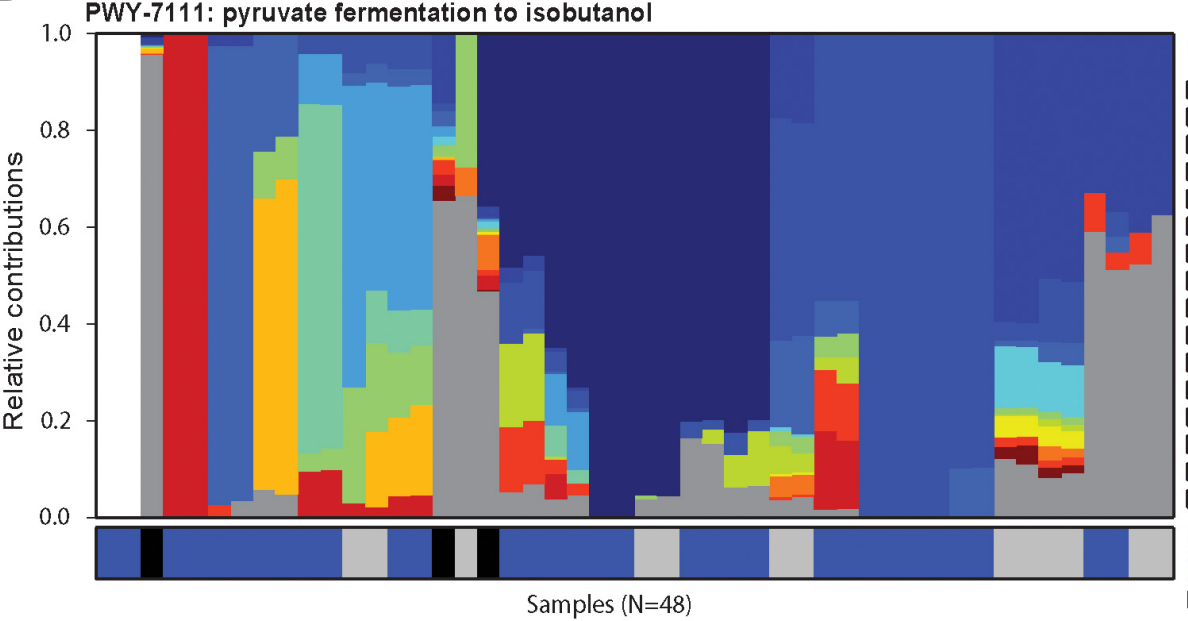

Stratifications:

Streptococcus mitis
Corynebacterium matruchotii
Rothia dentocariosa
Actinomyces viscosus
Streptococcus parasanguinis
Leptotrichia hofstadii
Streptococcus salivarius
Veillonella parvula
Rothia aeria
Neisseria flavescens
Actinomyces sp oral taxon 448
Neisseria sicca
Streptococcus cristatus
Streptococcus tigurinus
Leptotrichia buccalis
Other
Sample label:
Yost_2015
Jorth_2014
Duran-Pinedo_2015

FIGURE 5 | Contributors to pyruvate metabolism during disease. Relative contributions of different bacteria in the two pyruvate metabolism pathways in different samples. (A) Relative contributions of various organisms in the fermentation of pyruvate to acetate and lactate in each disease sample. Top 15 contributing organisms are listed by name and the rest are grouped as others. Columns are annotated by the study they come from. Different organisms perform similar functions in different disease samples maintaining the functional signature despite the community composition. (B) Relative contribution of various organisms in the fermentation of pyruvate to isobutanol. Again, functional signature is maintained despite differences in the organisms carrying out fermentation in the different samples.

The cobalamin riboswitch is the most predominant regulatory RNA identified (3\% of DE ncRNAs). Transcripts containing the cobalamin riboswitch from Tannerella and Porphyromonas (red complex), Veillonella (purple complex), and Corynebacterium (unaffiliated) are up-regulated, and transcripts from a species of Streptococcus of the yellow complex are down-regulated. These cobalamin riboswitches are associated with different genes in the different organisms. The riboswitch is found upstream of the 4-hydroxybutyryl-CoA dehydratase gene (adenosylcobalamin biosynthesis) in Tannerella; upstream of a hypothetical protein in Porphyromonas; upstream of methylmalonyl-CoA mutase large subunit gene (adenosylcobalamin dependent enzyme) in Veillonella; upstream of a transferase gene in Corynebacterium; and upstream of the ATP:cobalamin adenosyltransferase gene (adenosylcobalamin biosynthesis) in Streptococcus. Other riboswitches identified in the DE transcripts include the FMN, glycine, lysine, SAM, and the cyclic di-AMP riboswitch (Nelson et al., 2013). Apart from the ncRNAs listed above, many bacterial small RNAs (sRNAs) were also identified from a variety of genera in very low quantities. Various ribosomal protein regulatory elements were also more prevalent in the disease associated transcripts. These include the L10 leader from Fusobacterium sp.; the L17 downstream element from a yellow complex member of the genus Streptococcus; the L21 leader in an unaffiliated member of the Streptococcus genus; the S15 leader in Parvimonas sp.; and the L20 leader in Parvimonas sp., Veillonella sp., and a yellow 


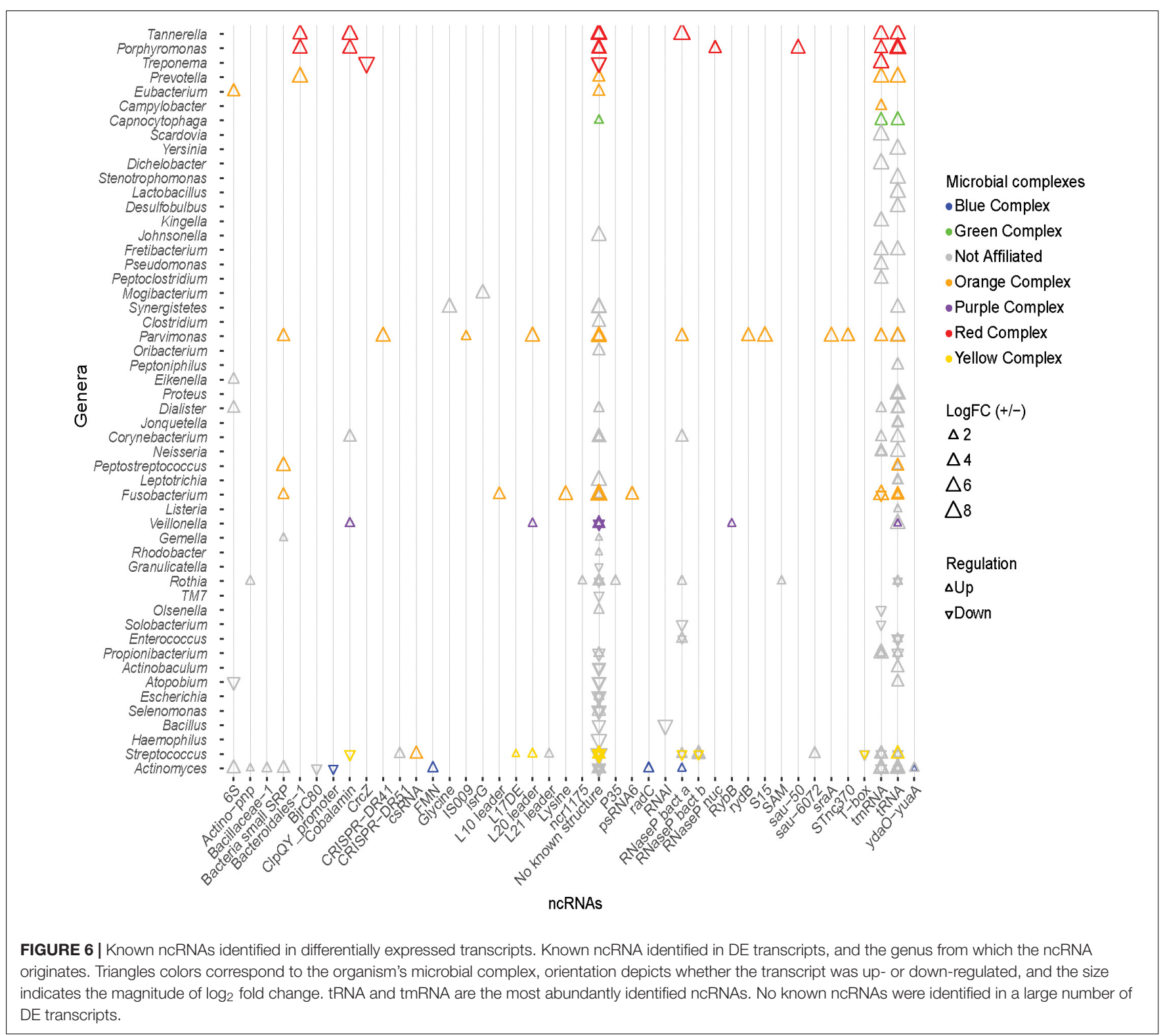

complex member of Streptococcus. Although a variety of known ncRNAs including riboswitches and small RNAs were identified in the DE transcripts, the majority of DE transcripts display no secondary structures of known function.

\section{Novel Putative Non-coding RNA in the DE Transcripts}

The large number of DE transcripts containing no previously described secondary structured RNA (Figure 6) were analyzed using GraphClust to discover novel putative ncRNA structures. This resulted in a total of 224 putative ncRNAs in the upregulated transcripts and 126 ncRNAs in the down-regulated transcripts. Each of these was manually curated to remove structures with minimal covariation in the predicted base pairing, or lacking a defined genomic context. Of these ncRNAs, 9 putative ncRNAs from the up-regulated and 6 putative ncRNAs from the down-regulated transcripts were scanned against the genomic database Refseq77 using cmsearch (Infernal 1.1.1) to identify additional homologs and determine the phylogenetic distribution of the putative regulatory element. Alignments for each of the 15 putative ncRNAs were analyzed using R-scape (Rivas et al., 2016a,b) to estimate statistical support for the predicted base pairs. Although the most of the putative ncRNAs were identified upstream of the same gene across taxa, like dihydroxyacetone kinase and the nitrogen fixation gene, many were found to be antisense upstream or downstream of the putatively regulated gene. A subset of the identified putative novel ncRNAs and their phylogenetic distribution are described below (Figure 7).

Of the 224-predicted putative ncRNAs in the up-regulated transcripts, 9 sense and antisense putative ncRNAs were chosen 


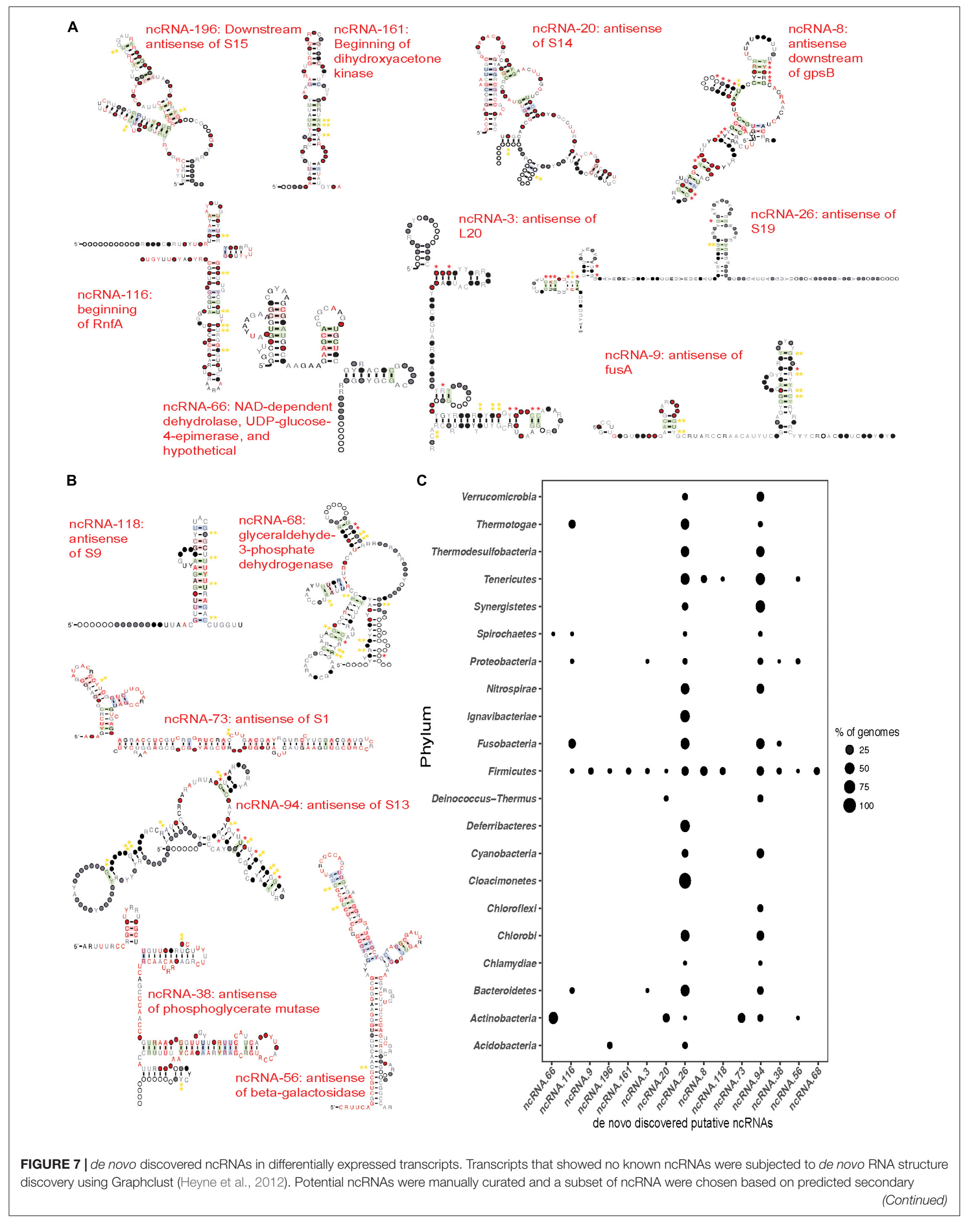




\section{FIGURE 7 | Continued}

structure and evidence for covariation in the base pairing and/or conserved genomic context. Significance of the covariation in predicted base pairs was further tested using R-scape (Rivas et al., 2016a,b). Red * indicate base pairs with $\mathrm{E} \leq 0.05$ and yellow ** indicate base pairs with $\mathrm{E} \leq 1$. (A) Novel putative ncRNAs discovered in the up-regulated transcripts. Consensus sequence and secondary structure of each ncRNA after scanning against Refseq77 and removing hits that did not share at least $60 \%$ of the secondary structure. Covarying base pairs are shaded. (B) Novel putative ncRNAs discovered in down-regulated transcripts produced as described above for the up-regulated transcripts. (C) Phylogenetic distribution of novel putative ncRNAs in the Bacterial domain. The points represent the percentage of genomes in which the ncRNA was found compared to the genomes in Refseq77 within a phylum. 13 of the 16 novel ncRNAs were found in a fraction of all the Firmicutes genomes. ncRNA-26 (antisense of ribosomal protein S19) and ncRNA-94 (antisense of ribosomal protein S13) are the most widely distributed across the Bacterial domain.

(Figure 7A) based on their secondary structure and genomic context (Supplementary File S1). Three of these ncRNAs appear to act as $5^{\prime}$-UTR cis regulators. The first, ncRNA-161, was identified in a transcript from Streptococcus anginosus CCUG 39159 ( $\log$ FC of $\sim 2.9$ ). This ncRNA is in the beginning of dihydroxyacetone kinase and is found only in the Firmicutes (Figures 7A,C), almost exclusively in Streptococcus with the exception of Bacillus sp. 1NLA3E. Our second candidate ncRNA116, was found upstream of the $r n f A$ gene in Fusobacterium nucleatum ATCC 25586. This nitrogen fixation gene involved in electron transport to nitrogenase (Schmehl et al., 1993) was found to have a fold change of $\sim 4.3$ between healthy and disease samples. Furthermore, the putative riboregulator is more highly distributed, appearing in a diverse group of phyla including Firmicutes, Proteobacteria, Bacteroidetes, Spirochaetes, Thermotogae, and Fusobacteria (Figure 7C). The third candidate, ncRNA-66, was identified upstream of a hypothetical protein in Actinomyces oral taxon 175 F0384 that had a logFC of $\sim 1.42$ and upstream of a hypothetical protein in Rothia dentocariosa ATCC 17931 with a $\log$ FC of $\sim 1.41$. Survey of the phylogenetic distribution revealed that ncRNA-66 was found distributed upstream of NAD-dependent dehydrolase, UDP-glucose-4-epimerase, and hypothetical proteins across the Actinobacteria and Spirochaetes (Figure 7C).

In addition to potential 5'UTR ncRNAs discussed above, our analyses also found four up-regulated putative cis-antisense regulators associated with ribosomal protein genes. Our first candidate, ncRNA-196, was identified in three up-regulated transcripts, located downstream and antisense of the ribosomal protein S15 coding region. Two of these transcripts belonged to strains of Rothia dentocariosa - M567 and ATCC 17931 with $\log$ FC of $\sim 2.35$ and 1.7, respectively, while the third transcript belonged to Streptococcus oral taxon $07173 \mathrm{H} 25 \mathrm{AP}$ displayed a $\log$ FC of 3.15. This putative ncRNA was found to be distributed through Firmicutes and Actinobacteria - mostly in the genus Streptococcus and in Bacillus megaterium WSH-002 within the Firmicutes; and Rothia dentocariosa in the Actinobacteria. Our second candidate, ncRNA-20 is another example of a putative cis antisense regulator of a ribosomal protein, S14. It was found in four up-regulated transcripts, three of which were from the genus Actinomyces and one from Rothia; Actinomyces oral taxon 175 F0384 displayed a $\log \mathrm{FC}$ of $\sim 2.21$; Actinomyces oris K20: 1.5; and Actinomyces naeslundii MG1: 1.5; Rothia dentocariosa M567: 1.8. Surveying Refseq77 revealed that this ncRNA is distributed across the Actinobacteria, DeinococcusThermus, and Firmicutes. A third candidate, ncRNA-3, is found cis-antisense of ribosomal protein L20 and represented in two transcripts, both from the genus Streptococcus - Streptococcus peroris ATCC 700780 and Streptococcus cristatus ATCC 51100 with a fold change of $\sim 1.69$ and $\sim 1.66$, respectively. Phylogenetic distribution of this ncRNA spans the Firmicutes, Proteobacteria, and Bacteroidetes. Finally, we identified a putative ncRNA antisense of ribosomal protein S19 (ncRNA-26). This ncRNA was found in three highly up-regulated transcripts from Tannerella forsythia ( $\log \mathrm{FC} \sim 7.5$ ), Fusobacterium nucleatum ( $\log \mathrm{FC} \sim 4.0$ ), and Veillonella parvula $(\log \mathrm{FC} \sim 2.3)$. It is widely distributed in the Bacterial domain. Of these antisense ncRNAs for ribosomal proteins, we find additional evidence for expression of ncRNA196 and ncRNA-3 in our recent study of the Streptococcus pneumoniae TIGR4 transcriptional profile (Warrier et al., 2018).

We also find up-regulated putative antisense ncRNAs associated with a variety of other processes. Our first candidate, ncRNA-9 is associated with the elongation factor G gene, fus $A$, that is differentially expressed in Streptococcus oligofermentans and Streptococcus anginosus. It is narrowly distributed and is identified only in $\sim 6 \%$ of the Firmicutes genomes in Refseq77. A second example is ncRNA-8, which is antisense and downstream of the cell cycle protein $g p s B$. We find ncRNA-8 in Streptococcus infantarius ATCC BAA-102 with the transcript having a $\log \mathrm{FC}$ of $\sim 2.5$. ncRNA- 8 is narrowly distributed to only Firmicutes and Tenericutes.

We also identified six promising antisense ncRNAs in the down-regulated transcripts (Figures 7B,C). Many of these also putatively regulate ribosomal proteins. ncRNA-118 was identified antisense of ribosomal protein S9 in Streptococcus mutans UA 159 (down-regulated by $\sim-1.48$ fold). This putative ncRNA is narrowly distributed across Refseq77 and is identified only in Firmicutes and Tenericutes (Figure 7C). A second example is ncRNA-73, which is antisense to the beginning of the ribosomal protein S1 coding region in Actinomyces oral taxon 180 F0310 (down-regulated $\sim-1.6$ fold). Surveying the bacterial genomes in Refseq77 revealed that this ncRNA is unique to the Actinobacteria. A third example is the putative ncRNA antisense of ribosomal protein S13, ncRNA-94, which was identified in Granulicatella adiacens ATCC 49175 (down-regulated -1.6 fold). ncRNA-94 is widely distributed across the Bacterial domain and is absent only in certain phyla such as Acidobacteria, Claocimonetes, and Deferribacteres.

In addition to ribosomal proteins, we also find down-regulated transcripts antisense to genes involved in sugar metabolism. ncRNA-56 was identified in the Klebsiella pneumoniae located antisense to the beginning of the beta-galactosidase gene, and is extremely down-regulated (-6.8 fold). Surveying its distribution, ncRNA-56 is found in the genomes of other Actinobacteria, 
Firmicutes, Proteobacteria, and Tenericutes. We also find two antisense ncRNAs putatively regulating different steps of glycolysis. ncRNA-38 was discovered antisense and overlapping the beginning of the down-regulated phosphoglycerate mutase gene. It was found in a fraction of the Firmicutes, Fusobacteria, and Proteobacteria (Figure 7C). The other putative regulator of glycolysis is ncRNA-68, which is found antisense and overlapping the translational start of glyceraldehyde-3-phosphate dehydrogenase. However, ncRNA-68 was found only in the Firmicutes. By applying de novo discovery pipelines on the DE transcripts, we have identified several promising sense and antisense putative regulators of bacterial ribosomal proteins and other metabolic genes that are associated with periodontitis as reflected in their expression in the healthy vs. disease samples.

\section{DISCUSSION}

Here we present a meta study of three existing sets of metagenomes and metatranscriptomes (Duran-Pinedo et al., 2014; Jorth et al., 2014; Yost et al., 2015) related to oral health and disease to identify commonality in the progression of periodontitis. Although more commonly performed with multiple metagenomes, combined metatranscriptomics analyses enable us to increase statistical support for the findings that are made, as well as understand the commonalities between different studies. From our analyses we find that nearly $~ 50 \%$ of DE transcripts were from bacteria not previously classified into disease associated complexes (Figure 1), and only $20 \%$ originate from organisms of the red and orange complexes. This mimics previous findings that showed putative virulence factors were upregulated in larger numbers of bacteria that did not belong to the red or orange complexes (Yost et al., 2015). However, despite the small number of DE transcripts originating from the members of the red and orange complexes, these transcripts show the greatest magnitude of up- or down-regulation.

Healthy samples do not cluster based on the transcript expression, and this is not surprising since mature communities are extremely diverse and often show variations between individual sites within the oral cavity (Marsh, 2006). However, similar expression patterns are also not observed across the disease samples. This lack in correlation among the disease samples is somewhat surprising since previous work suggested that the disease associated microbiota are more similar than health associated communities (Jorth et al., 2014). In this case such differences may also be attributed to differences in sample inclusion criteria and collection sites, batch effects, and methodological biases, of individual studies. However, these are unlikely to be the only reasons for such lack in correlation. Meta-analyses of RNA-seq data from four studies comprising of 6-13 tissues each from 11 vertebrate species using similar cross sample normalization methods revealed clustering of samples by tissue rather than study or species (Sudmant et al., 2015). This suggests that true commonality between studies can be inferred by these meta-analyses despite any batch effects and methodological biases that might exist. To support this, when comparing individual datasets, no single organism, even at the genus level, was functionally active in all the disease samples suggesting inherent variability between communities. Our analyses also find highly active viruses in the periodontal pocket (Figure 3A) of many disease samples. However, all but the Mastadenovirus are plant related and may reflect the individual's diet during the sampling. This observed large variety in the functionally active bacterial community composition supports the polymicrobial nature of periodontitis.

Previous metatranscriptomics analyses identified a metabolic shift in disease associated communities toward increased nucleotide biosynthesis, iron acquisition, cobalamin transport, and fermentation of lysine, histidine, and pyruvate (Jorth et al., 2014; Yost et al., 2015). Our own GO term enrichment analyses from the pooled datasets mimics these findings suggesting that these functional shifts are common during the progression of periodontitis. Further exploration of the differentially expressed steps along the pyruvate metabolism pathways using KEGG Orthology showed that different enzymes are differentially expressed in diverse organisms. Even within individual metatranscriptomes, we identified a large degree of diversity in the organisms contributing to pyruvate fermentation (Figures 4, 5A,B). Thus, our analysis supports a model where a variety of different bacteria may drive the metabolic process, supporting the polymicrobial dysbiotic nature of the periodontal disease (Darveau, 2010; Hajishengallis and Lamont, 2012; Rosier et al., 2014; Szafrański et al., 2015). Furthermore, our findings also support the notion that periodontitis occurs despite idiosyncratic differences between individuals as long as the community undergoes a switch in its functional and metabolic signals (Dabdoub et al., 2016).

Bacterial metabolic processes are finely controlled. Bacterial non-coding RNAs (ncRNAs) modulate posttranscriptional gene expression genes in response to environmental cues (Breaker, 2012) using a variety of mechanisms including: impacting mRNA decay rate (Desnoyers et al., 2013), regulating translation initiation (Urban and Vogel, 2007; Frohlich and Vogel, 2009), and biosynthesis of ribosomal proteins (Deiorio-Haggar et al., 2013). Given the switch in the functional and metabolic signal during periodontal disease, we searched for putative ncRNAs to drive such changes. Surveying the DE transcripts for known ncRNAs revealed trends similar to those previously observed (Duran-Pinedo et al., 2015) with tRNAs and tmRNAs the most abundant ncRNAs identified in the DE transcripts (Figure 6). We also identified several other ncRNAs including 6S, bacterial small signal recognition particle, and the cobalamin, FMN, glycine, lysine, and SAM riboswitches, ribosomal protein leaders, and bacterial RNase $\mathrm{P}$ class $\mathrm{A}$ and $\mathrm{B}$. However, no known ncRNAs were identified in a large majority of DE transcripts.

De novo discovery of ncRNAs in DE transcripts lacking known ncRNAs revealed many novel, putative structured elements. Manual curation of these revealed promising ncRNAs identified sense and antisense to coding regions. Some of the promising ncRNAs and their phylogenetic distributions are presented in Figure 7, several of which have many predicted basepairs with statistical support. We find putative sense ncRNAs upstream or near the $5^{\prime}$-end of metabolic genes and antisense ncRNAs corresponding to metabolic genes and many ribosomal 
protein genes including S1, S9, S13, S14, S14, S19, and L20. Despite a suite of well-characterized RNA cis-regulators for ribosomal protein genes (Deiorio-Haggar et al., 2013; Fu et al., 2013, 2014; Slinger et al., 2014; Babina et al., 2015), remarkably there is a dearth of evidence for wide-spread antisense regulation. Only two examples have been described to our knowledge: a $s^{\mathrm{B}}$ induced (stress-induced) transcript has been identified in $B$. subtilis that is anti-sense to $r p s D$, resulting in downregulation of $r p s D$ transcript and presumably S4 expression (Mars et al., 2015), and an antisense ncRNA spanning 14 genes of a ribosomal protein operon protects the transcript by hiding RNase E sites providing the Prochlorococcus MED4 RNA an enhanced half-life during phage infection (Stazic et al., 2011). The seven putative antisense ncRNA we discovered are likely novel regulators of bacterial ribosomal proteins that might conform to the aforementioned mechanisms of actions or have completely novel mechanisms but are likely worthy of further study in the future as potential targets for antimicrobials treating or preventing periodontitis. This combination of known and novel putative ncRNAs regulating metabolic processes and translational machinery likely facilitate the progression of the disease.

\section{DATA AVAILABILITY STATEMENT}

Publicly available datasets were analyzed in this study. This data can be found here: SRP033605, HOMD: 20130522, HOMD: 20141024.

\section{REFERENCES}

Abubucker, S., Segata, N., Goll, J., Schubert, A. M., Izard, J., Cantarel, B. L., et al. (2012). Metabolic reconstruction for metagenomic data and its application to the human microbiome. PLoS Comput. Biol. 8:e1002358. doi: 10.1371/journal. pcbi. 1002358

Abusleme, L., Dupuy, A. K., Dutzan, N., Silva, N., Burleson, J. A., Strausbaugh, L. D., et al. (2013). The subgingival microbiome in health and periodontitis and its relationship with community biomass and inflammation. ISME J. 7, 1016-1025. doi: 10.1038/ismej.2012.174

Ai, D., Huang, R., Wen, J., Li, C., Zhu, J., and Xia, L. C. (2017). Integrated metagenomic data analysis demonstrates that a loss of diversity in oral microbiota is associated with periodontitis. BMC Genomics 18:1041. doi: 10 . 1186/s12864-016-3254-5

Altschul, S. F., Gish, W., Miller, W., Myers, E. W., and Lipman, D. J. (1990). Basic local alignment search tool. J. Mol. Biol. 215, 403-410. doi: 10.1016/S0022-2836(05)80360-2S0022-2836(05) 80360-2

Ashimoto, A., Chen, C., Bakker, I., and Slots, J. (1996). Polymerase chain reaction detection of 8 putative periodontal pathogens in subgingival plaque of gingivitis and advanced periodontitis lesions. Oral. Microbiol. Immunol. 11, 266-273. doi: 10.1111/j.1399-302X.1996.tb00180.x

Babina, A. M., Soo, M. W., Fu, Y., and Meyer, M. M. (2015). An S6:S18 complex inhibits translation of E. coli rpsF. RNA 21, 2039-2046. doi: 10.1261/rna.049544. 115

Breaker, R. R. (2012). Riboswitches and the RNA world. Cold Spring Harb. Perspect. Biol. 4, a003566. doi: 10.1101/cshperspect.a003566

Burge, S. W., Daub, J., Eberhardt, R., Tate, J., Barquist, L., Nawrocki, E. P., et al. (2013). Rfam 11.0: 10 years of RNA families. Nucleic Acids Res. 41, D226-D232. doi: $10.1093 / \mathrm{nar} / \mathrm{gks} 1005$

\section{AUTHOR CONTRIBUTIONS}

MM conceived the study and edited the manuscript. NR-M performed all the analyses and wrote the manuscript. Both authors read and approved the final manuscript.

\section{FUNDING}

This work was supported by an NIH grant (R21DE025051) to MM.

\section{ACKNOWLEDGMENTS}

The authors would like to thank Jon Anthony for the setup of many of the tools used and Matt Crum for useful discussions and valuable assistance.

\section{SUPPLEMENTARY MATERIAL}

The Supplementary Material for this article can be found online at: https://www.frontiersin.org/articles/10.3389/fmicb. 2020.00482/full\#supplementary-material

TABLE S1 | List of differentially expressed transcripts estimated form the pooled dataset spanning 48 healthy and 49 disease samples. A list of 859 DE transcripts with $\log \mathrm{FC}>1$ or $\log \mathrm{FC}<-1$ and $p<0.05$.

FILE S1 | Alignment files for the discovered novel putative ncRNAs.

Dabdoub, S. M., Ganesan, S. M., and Kumar, P. S. (2016). Comparative metagenomics reveals taxonomically idiosyncratic yet functionally congruent communities in periodontitis. Sci. Rep 6, 38993. doi: 10.1038/srep38993

Darveau, R. P. (2010). Periodontitis: a polymicrobial disruption of host homeostasis. Nat. Rev. Microbiol. 8, 481-490. doi: 10.1038/nrmicro2337

Darveau, R. P., Hajishengallis, G., and Curtis, M. A. (2012). Porphyromonas gingivalis as a potential community activist for disease. J. Dent. Res. 91, 816-820. doi: $10.1177 / 0022034512453589$

Daub, J., Gardner, P. P., Tate, J., Ramskold, D., Manske, M., Scott, W. G., et al. (2008). The RNA WikiProject: community annotation of RNA families. RNA 14, 2462-2464. doi: 10.1261/rna.1200508

Deiorio-Haggar, K., Anthony, J., and Meyer, M. M. (2013). RNA structures regulating ribosomal protein biosynthesis in bacilli. RNA Biol. 10, 1180-1184. doi: $10.4161 /$ rna.24151

Desnoyers, G., Bouchard, M. P., and Massé, E. (2013). New insights into small RNA-dependent translational regulation in prokaryotes. Trends Genet. 29, 92-98. doi: 10.1016/j.tig.2012.10.004

Duran-Pinedo, A. E., Chen, T., Teles, R., Starr, J. R., Wang, X., Krishnan, K., et al. (2014). Community-wide transcriptome of the oral microbiome in subjects with and without periodontitis. ISME J. 8, 1659-1672. doi: 10.1038/ismej.2014. 23

Duran-Pinedo, A. E., Yost, S., and Frias-Lopez, J. (2015). Small RNA transcriptome of the oral microbiome during periodontitis progression. Appl. Environ. Microbiol. 81, 6688-6699. doi: 10.1128/AEM.01782-15

Finn, R. D., Bateman, A., Clements, J., Coggill, P., Eberhardt, R. Y., Eddy, S. R., et al. (2014). Pfam: the protein families database. Nucleic Acids Res. 42, D222-D230. doi: $10.1093 /$ nar/gkt1223

Finn, R. D., Clements, J., and Eddy, S. R. (2011). HMMER web server: interactive sequence similarity searching. Nucleic Acids Res. 39, W29-W37. doi: 10.1093/ nar/gkr367 
Frohlich, K. S., and Vogel, J. (2009). Activation of gene expression by small RNA. Curr. Opin. Microbiol. 12, 674-682. doi: 10.1016/j.mib.2009.09.009

Fu, Y., Deiorio-Haggar, K., Anthony, J., and Meyer, M. M. (2013). Most RNAs regulating ribosomal protein biosynthesis in Escherichia coli are narrowly distributed to Gammaproteobacteria. Nucleic Acids Res. 41, 3491-3503. doi: 10.1093/nar/gkt055

Fu, Y., Deiorio-Haggar, K., Soo, M. W., and Meyer, M. M. (2014). Bacterial RNA motif in the $5^{\prime}$ UTR of rpsF interacts with an S6:S18 complex. RNA 20, 168-176. doi: 10.1261/rna.041285.113

Gibbons, S. M., Duvallet, C., and Alm, E. J. (2018). Correcting for batch effects in case-control microbiome studies. PLoS Comput. Biol. 14:e1006102. doi: 10. 1371/journal.pcbi.1006102

Grabherr, M. G., Haas, B. J., Yassour, M., Levin, J. Z., Thompson, D. A., Amit, I., et al. (2011). Full-length transcriptome assembly from RNA-Seq data without a reference genome. Nat. Biotechnol. 29, 644-652. doi: 10.1038/nbt.1883

Griffiths-Jones, S. (2005). RALEE - RNA alignment editor in Emacs. Bioinformatics 21, 257-259. doi: 10.1093/bioinformatics/bth489

Griffiths-Jones, S., Bateman, A., Marshall, M., Khanna, A., and Eddy, S. R. (2003). Rfam: an RNA family database. Nucleic Acids Res. 31, 439-441. doi: 10.1093/ nar/gkg006

Griffiths-Jones, S., Moxon, S., Marshall, M., Khanna, A., Eddy, S. R., and Bateman, A. (2005). Rfam: annotating non-coding RNAs in complete genomes. Nucleic Acids Res. 33, D121-D124. doi: 10.1093/nar/gki081

Gruber, A. R., Findeiß, S., Washietl, S., Hofacker, I. L., and Stadler, P. F. (2010). RNAZ 2.0: improved noncoding RNA detection. Pacific Symp. Biocomput. 69-79. doi: 10.1142/9789814295291_0009

Haas, B. J., Papanicolaou, A., Yassour, M., Grabherr, M., Blood, P. D., Bowden, J., et al. (2013). De novo transcript sequence reconstruction from RNA-seq using the Trinity platform for reference generation and analysis. Nat. Protoc. 8, 1494-1512. doi: 10.1038/nprot.2013.084

Hajishengallis, G., Darveau, R. P., and Curtis, M. A. (2012). The keystone-pathogen hypothesis. Nat. Rev. Microbiol. 10, 717-725. doi: 10.1038/nrmicro2873

Hajishengallis, G., and Lamont, R. J. (2012). Beyond the red complex and into more complexity: the polymicrobial synergy and dysbiosis (PSD) model of periodontal disease etiology. Mol. Oral Microbiol. 27, 409-419. doi: 10.1111/j. 2041-1014.2012.00663.x

Hajishengallis, G., Liang, S., Payne, M. A., Hashim, A., Jotwani, R., Eskan, M. A., et al. (2011). Low-abundance biofilm species orchestrates inflammatory periodontal disease through the commensal microbiota and complement. Cell Host Microbe 10, 497-506. doi: 10.1016/j.chom.2011.10.006

Heyne, S., Costa, F., Rose, D., and Backofen, R. (2012). Graphclust: alignmentfree structural clustering of local RNA secondary structures. Bioinformatics 28, i224-i232. doi: 10.1093/bioinformatics/bts224

Holt, S. C., and Ebersole, J. L. (2005). Porphyromonas gingivalis, Treponema denticola, and Tannerella forsythia: the "red complex", a prototype polybacterial pathogenic consortium in periodontitis. Periodontol 2000, 72-122. doi: 10.1111/ j.1600-0757.2005.00113.x

Jorth, P., Turner, K. H., Gumus, P., Nizam, N., Buduneli, N., and Whiteley, M. (2014). Metatranscriptomics of the human oral microbiome during health and disease. MBio 5:e01012-14. doi: 10.1128/mBio.01012-14

Kumar, P. S., Griffen, A. L., Barton, J. A., Paster, B. J., Moeschberger, M. L., and Leys, E. J. (2003). New bacterial species associated with chronic periodontitis. J. Dent. Res. 82, 338-344. doi: 10.1177/154405910308200503

Lagesen, K., Hallin, P., Rødland, E. A., Stærfeldt, H. H., Rognes, T., and Ussery, D. W. (2007). RNAmmer: consistent and rapid annotation of ribosomal RNA genes. Nucleic Acids Res. 35, 3100-3108. doi: 10.1093/nar/gkm160

Lamont, R. J., and Hajishengallis, G. (2015). Polymicrobial synergy and dysbiosis in inflammatory disease. Trends Mol. Med. 21, 172-183. doi: 10.1016/j.molmed. 2014.11.004

Langmead, B., Salzberg, S. L., and Langmead . (2013). Fast Gapped-Read Alignment With Bowtie2. Nat. Methods 9, 357-359. doi: 10.1038/nmeth.1923. Fast

Lorenz, R., Bernhart, S. H., Höner Zu Siederdissen, C., Tafer, H., Flamm, C., Stadler, P. F., et al. (2011). ViennaRNA Package 2.0. Algorithms Mol. Biol. 6:26. doi: 10.1186/1748-7188-6-26

Luo, W., and Brouwer, C. (2013). Pathview: an R/Bioconductor package for pathway-based data integration and visualization. Bioinformatics 29, 18301831. doi: 10.1093/bioinformatics/btt285
Luo, W., and Brouwer, C. (2015). Pathview: pathway based data integration and visualization. Bioinformatics 29, 1830-1831.

Mars, R. A. T., Mendonça, K., Denham, E. L., and van Dijl, J. M. (2015). The reduction in small ribosomal subunit abundance in ethanol-stressed cells of Bacillus subtilis is mediated by a SigB-dependent antisense RNA. Biochim. Biophys. Acta Mol. Cell Res. 1853(10 Pt A), 2553-2559. doi: 10.1016/j.bbamcr. 2015.06.009

Marsh, P. D. (2006). Dental diseases-are these examples of ecological catastrophes? Int. J. Dent. Hyg. 4(Suppl. 1), 3-10. doi: 10.1111/j.1601-5037.2006.00195.x

Moriya, Y., Itoh, M., Okuda, S., Yoshizawa, A. C., and Kanehisa, M. (2007). KAAS: an automatic genome annotation and pathway reconstruction server. Nucleic Acids Res. 35, W182-W185. doi: 10.1093/nar/gkm321

Nawrocki, E. P., Burge, S. W., Bateman, A., Daub, J., Eberhardt, R. Y., Eddy, S. R., et al. (2015). Rfam 12.0: updates to the RNA families database. Nucleic Acids Res. 43, D130-D137. doi: 10.1093/nar/gku1063

Nawrocki, E. P., and Eddy, S. R. (2013). Infernal 1.1: 100-fold faster RNA homology searches. Bioinformatics 29, 2933-2935. doi: 10.1093/bioinformatics/btt509

Nawrocki, E. P., Kolbe, D. L., and Eddy, S. R. (2009). Infernal 1.0: inference of RNA alignments. Bioinformatics 25, 1335-1337. doi: 10.1093/bioinformatics/btp157

Nelson, J. W., Sudarsan, N., Furukawa, K., Weinberg, Z., Wang, J. X., and Breaker, R. R. (2013). Riboswitches in eubacteria sense the second messenger c-di-AMP. Nat. Chem. Biol. 9, 834-839. doi: 10.1038/nchembio.1363

Neufing, P. J., Shearwin, K. E., and Egan, J. B. (2001). Establishing lysogenic transcription in the temperate coliphage 186. J. Bacteriol. 183, 2376-2379. doi: 10.1128/JB.183.7.2376-2379.2001

O'Leary, N. A., Wright, M. W., Brister, J. R., Ciufo, S., Haddad, D., McVeigh, R., et al. (2016). Reference sequence (RefSeq) database at NCBI: current status, taxonomic expansion, and functional annotation. Nucleic Acids Res. 44, D733D745. doi: 10.1093/nar/gkv1189

Orth, R. K. H., O’Brien-Simpson, N. M., Dashper, S. G., and Reynolds, E. C. (2011). Synergistic virulence of Porphyromonas gingivalis and Treponema denticola in a murine periodontitis model. Mol. Oral Microbiol. 26, 229-240. doi: 10.1111/j. 2041-1014.2011.00612.x

Patro, R., Duggal, G., and Kingsford, C. (2015). Salmon: accurate, versatile and ultrafast quantification from RNA-seq data using lightweight-alignment. bioRxiv [preprint]. doi: 10.1101/021592

R Core Team (2015). R Core Team. R A Lang. Environ. Stat. Comput. R Found. Stat. Comput. Vienna: R Core Team.

Rivas, E., Clements, J., and Eddy, S. R. (2016a). A statistical test for conserved RNA structure shows lack of evidence for structure in lncRNAs. Nat. Methods 14, 45-48. doi: 10.1038/nmeth.4066

Rivas, E., Clements, J., and Eddy, S. R. (2016b). Lack of evidence for conserved secondary structure in long noncoding RNAs. Nat. Methods 14, 45-48.

Robinson, M. D., McCarthy, D. J., and Smyth, G. K. (2009). edgeR: a bioconductor package for differential expression analysis of digital gene expression data. Bioinformatics 26, 139-140. doi: 10.1093/bioinformatics/ btp616

Rosier, B. T., De Jager, M., Zaura, E., and Krom, B. P. (2014). Historical and contemporary hypotheses on the development of oral diseases: are we there yet? Front. Cell. Infect. Microbiol. 4:92. doi: 10.3389/fcimb.2014. 00092

Sayed, N., Jousselin, A., and Felden, B. (2012). A cis-antisense RNA acts in trans in Staphylococcus aureus to control translation of a human cytolytic peptide. Nat. Struct. Mol. Biol. 19, 105-112. doi: 10.1038/nsmb.2193

Schmehl, M., Jahn, A., Meyer zu Vilsendorf, A., Hennecke, S., Masepohl, B., Schuppler, M., et al. (1993). Identification of a new class of nitrogen fixation genes in Rhodobacter capsalatus: a putative membrane complex involved in electron transport to nitrogenase. MGG Mol. Gen. Genet. 241, 602-615. doi: 10.1007/BF00279903

Slinger, B. L., Deiorio-Haggar, K., Anthony, J. S., Gilligan, M. M., and Meyer, M. M. (2014). Discovery and validation of novel and distinct RNA regulators for ribosomal protein S15 in diverse bacterial phyla. BMC Genomics 15:657. doi: 10.1186/1471-2164-15-657

Slots, J. (1977). The predominant cultivable microflora of advanced periodontitis. Eur. J. Oral Sci. 85, 114-121. doi: 10.1111/j.1600-0722.1977.tb00541.x

Smith, C., Heyne, S., Richter, A. S., Will, S., and Backofen, R. (2010). Freiburg RNA Tools: a web server integrating IntaRNA, ExpaRNA and LocARNA. Nucleic Acids Res. 38, W373-W377. doi: 10.1093/nar/gkq316 
Smith-Unna, R., Boursnell, C., Patro, R., Hibberd, J. M., and Kelly, S. (2016). TransRate: reference-free quality assessment of de novo transcriptome assemblies. Genome Res. 26, 1134-1144. doi: 10.1101/gr.196469.115

Socransky, S. S., and Haffajee, A. D. (2005). Periodontal microbial ecology. Periodontol 2000, 135-187. doi: 10.1111/j.1600-0757.2005.00107.x

Socransky, S. S., Haffajee, A. D., Cugini, M. A., Smith, C., and Kent, R. L. (1998). Microbial complexes in subgingival plaque. J. Clin. Periodontol. 25, 134-144. doi: 10.1111/j.1600-051X.1998.tb02419.x

Stazic, D., Lindell, D., and Steglich, C. (2011). Antisense RNA protects mRNA from RNase e degradation by RNA-RNA duplex formation during phage infection. Nucleic Acids Res. 39, 4890-4899. doi: 10.1093/nar/gkr037

Steffen, P., Voß, B., Rehmsmeier, M., Reeder, J., and Giegerich, R. (2006). RNAshapes: an integrated RNA analysis package based on abstract shapes. Bioinformatics 22, 500-503. doi: 10.1093/bioinformatics/btk010

Sudmant, P. H., Alexis, M. S., and Burge, C. B. (2015). Meta-analysis of RNAseq expression data across species, tissues and studies. Genome Biol. 16:287. doi: 10.1186/s13059-015-0853-4

Szafrański, S. P., Deng, Z.-L., Tomasch, J., Jarek, M., Bhuju, S., Meisinger, C., et al. (2015). Functional biomarkers for chronic periodontitis and insights into the roles of Prevotella nigrescens and Fusobacterium nucleatum; a metatranscriptome analysis. Npj Biofilms Microbiomes 1:15017. doi: 10.1038/ npjbiofilms.2015.17

Truong, D. T., Franzosa, E. A., Tickle, T. L., Scholz, M., Weingart, G., Pasolli, E., et al. (2015). MetaPhlAn2 for enhanced metagenomic taxonomic profiling. Nat. Methods 12, 902-903. doi: 10.1038/nmeth.3589

Urban, J. H., and Vogel, J. (2007). Translational control and target recognition by Escherichia coli small RNAs in vivo. Nucleic Acids Res. 35, 1018-1037. doi: 10.1093/nar/gkl1040

Wang, J., Qi, J., Zhao, H., He, S., Zhang, Y., Wei, S., et al. (2013). Metagenomic sequencing reveals microbiota and its functional potential associated with periodontal disease. Sci. Rep. 3:1843. doi: 10.1038/srep 01843
Warrier, I., Ram-Mohan, N., Zhu, Z., Hazery, A., Echlin, H., Rosch, J., et al. (2018). The Transcriptional landscape of Streptococcus pneumoniae TIGR4 reveals a complex operon architecture and abundant riboregulation critical for growth and virulence. PLoS Pathog. 14:e1007461. doi: 10.1371/journal.ppat.1007461

Will, S., Joshi, T., Hofacker, I. L., Stadler, P. F., and Backofen, R. (2012). LocARNAP: accurate boundary prediction and improved detection of structural RNAs. RNA 18, 900-914. doi: 10.1261/rna.029041.111

Will, S., Reiche, K., Hofacker, I. L., Stadler, P. F., and Backofen, R. (2007). Inferring noncoding RNA families and classes by means of genome-scale structure-based clustering. PLoS Comput. Biol. 3:e65. doi: 10.1371/journal.pcbi.0030065

Ximénez-Fyvie, L. A., Haffajee, A. D., and Socransky, S. S. (2000). Microbial composition of supra- and subgingival plaque in subjects with adult periodontitis. J. Clin. Periodontol. 27, 722-732. doi: 10.1034/j.1600-051x.2000. 027010722.x

Yost, S., Duran-Pinedo, A. E., Teles, R., Krishnan, K., and Frias-Lopez, J. (2015). Functional signatures of oral dysbiosis during periodontitis progression revealed by microbial metatranscriptome analysis. Genome Med. 7:27. doi: 10 . 1186/s13073-015-0153-3

Young, M. D., Wakefield, M. J., Smyth, G. K., and Oshlack, A. (2010). Gene ontology analysis for RNA-seq: accounting for selection bias. Genome Biol. 11:R14. doi: 10.1186/gb-2010-11-2-r14

Conflict of Interest: The authors declare that the research was conducted in the absence of any commercial or financial relationships that could be construed as a potential conflict of interest.

Copyright (c) 2020 Ram-Mohan and Meyer. This is an open-access article distributed under the terms of the Creative Commons Attribution License (CC BY). The use, distribution or reproduction in other forums is permitted, provided the original author(s) and the copyright owner(s) are credited and that the original publication in this journal is cited, in accordance with accepted academic practice. No use, distribution or reproduction is permitted which does not comply with these terms. 